\title{
Low Complex Adaptive Fast Converged Kalman Filter for Cardiac ECG Artifacts Elimination
}

\author{
Y. Murali Krishna ${ }^{1}$, N.Sayedu Khasim ${ }^{2}$ and V.Naveen Raja ${ }^{3}$ \\ ${ }^{1,2,3}$ Dept. of Electronics and Communication Engineering,Narasaraopeta Engineering \\ College,Narasaraopet,AP,India \\ ${ }^{1}$ yadavalli437@gmail.com, ${ }^{2}$ sayedukhasim@gmail.com, \\ ${ }^{3}$ naveenraja.velchuri@gmail.com
}

\begin{abstract}
The aim of this paper is to reduce the main problems in biomedical data processing like electrocardiography is the separation of the wanted signal from noises caused by power line interference, external electromagnetic fields, random body movements and respiration. Different types of digital filters are used to remove signal components from unwanted frequency ranges. It is difficult to apply filters with fixed coefficients to reduce Biomedical Signal noises, because human behavior is not exact known depending on the time. Adaptive filter technique is required to overcome this problem. In this paper different types of adaptive filters are considered to reduce the ECG signal noises like Base Line Interference, EM noise and muscle artifact, results of simulations in MATLAB are presented. While the LMS algorithm and its normalized version (NLMS), have been thoroughly used and studied. Connections between the Kalman filter and the RLS algorithm have been established however, the connection between the Kalman filter and the LMS algorithm has not received much attention. By linking these two algorithms, a new normalized Kalman based LMS (KLMS) algorithm can be derived that has some advantages to the classical one. Their stability is guaranteed since they are a special case of the Kalman filter. More, they suggests a new way to control the step size, that results in good convergence properties for a large range of input signal powers, that occur in many applications. In this paper, different algorithms based on the correlation form, information form (IKLMS) and simplified versions (SIKLMS) of these are presented. The simplified form maintains the good convergence properties of the KLMS with slightly lower computational complexity.
\end{abstract}

Keywords: ECG Signal, Adaptive Algorithms, Base Line Interferences, EM noise, MA noise

\section{Introduction}

ECG is Obtained by recordings of noninvasive technique is a harmless, safe, and quick method of cardiovascular diagnosis. The main issue in ECG is the extraction of high-resolution ECG signals from recordings with background noise

The aim for ECG signal enhancement is to separate the valid signal components from the undesired artifacts which causes damage to the recordings. Several have been reported in this paper to address ECG enhancement using adaptive filters, which permit to detect time varying potentials and to track the dynamic variations of the signals. In proposed an RLS based adaptive recurrent filter to acquire the impulse response of normal QRS complexes, and then applied it for arrhythmia detection in ambulatory ECG recordings. 
In this paper, the LMS algorithm operates on an instantaneous basis such that the weight vector is updated every new sample within the occurrence, based on an instantaneous gradient estimate. The reference inputs to the basic adaptive LMS algorithm are deterministic functions and are defined by a periodically extended, truncated set of orthonormal basis functions.

There are certain clinical applications of ECG signal processing that require adaptive filters with large number of taps. In such applications the conventional LMS algorithm is computationally expensive to implement The LMS algorithm and NLMS (normalized LMS) algorithm requires few computations. However, there is a strong need to improve the convergence speed of the LMS and NLMS algorithms.

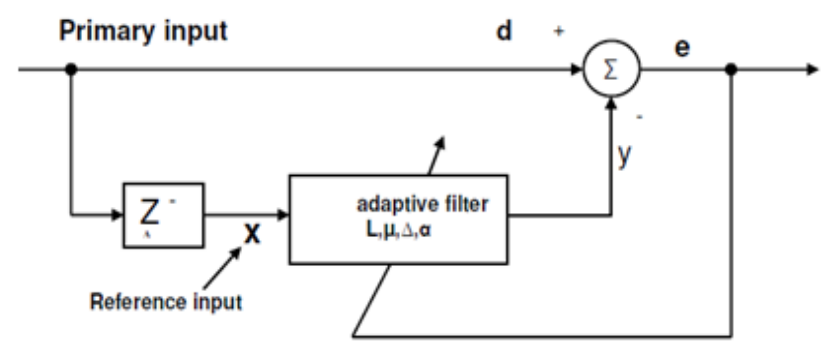

Figure 1. Adaptive Filter for Noise Cancellation

In practice, LMS is replaced with its Normalized version, NLMS. In practical applications of LMS filtering, a key parameter is the step size. If the step size is large, the convergence rate of the LMS algorithm will be rapid, but the steady-state mean square error (MSE) will increase. On the other hand, if the step size is small, the steady state MSE will be small, but the convergence rate will be slow. Thus, the step size provides a tradeoff between the convergence rate and the steady-state MSE of the LMS algorithm. The Least Mean Squares (LMS) algorithm for adaptive filters has been extensively studied and tested in a broad range of applications [1]-[3]. In [4] a relation between the Recursive Least Squares (RLS) and the Kalman filter [11] algorithm is determined, and in [10] the tracking convergence of the LMS, RLS and extended RLS algorithms, based on the Kalman filter, are compared. However, there is no link established between the Kalman filter and the LMS algorithm. In this paper we used the combination of LMS and Kalman filter to implement ANCs for ECG denoising. Furthermore some simplified versions of KLMS are also implemented. These are Information form KLMS (IKLMS) and Simplified IKLMS (SIKLMS). To study the performance of the proposed algorithm to effectively remove the noise from the ECG signal, we carried out simulations on MIT-BIH database for different artifacts.

\section{The LMS Adaptation Algorithm}

The LMS (least mean squares) algorithm is an approximation of the steepest descent algorithm which uses an instantaneous estimate of the gradient vector of a cost function [5]. The estimate of the gradient is based on sample values of the tap-input vector and an error signal. The algorithm iterates over each coefficient in the filter, moving it in the direction of the approximated gradient. For the LMS algorithm it is necessary to have a reference signal $d[n]$ representing the desired filter output. The difference 
between the reference signal and the actual output of the transversal filter is the error signal

$$
e(n)=d(n)-h(n) x(n)
$$

The task of the LMS algorithm is to find a set of filter coefficients $c$ that minimize the expected value of the quadratic error signal, i.e., to achieve the least mean squared error. The squared error and its expected value are (for simplicity of notation and perception we drop the dependence of all variables on time n. In the LMS algorithm, however, a very short-term estimate is used by only taking into account the current samples. Here, we introduced the 'step-size' parameter $\mu$, which controls the distance we move along the error surface. In the LMS algorithm the update of the coefficients is performed at every time instant $n$

$$
h(t+1)=h(t)+\mu e^{*}(n) x(n)
$$

The 'step-size' parameter $\mu$ introduced in above equation controls how far we move along the error function surface at each update step. $\mu$ certainly has to be chosen $\mu>0$ (otherwise we would move the coefficient vector in a direction towards larger squared error). Closer analysis reveals, that the upper bound for $\mu$ for stable behavior of the LMS algorithm depends on the largest eigenvalue $\mu_{\max }$ of the tap-input auto-correlation matrix $\mathrm{R}$ and thus on the input signal. For stable adaptation behavior the step-size has to be

$$
\mu=2 / \mu_{\max }
$$

The NLMS algorithm has been developed from different viewpoints, such as Goodwin and Sin demonstrate the formulation from the constrain optimization problem by the method of Lagrange multipliers. The NLMS exhibited a significant improvement over the LMS in convergence rate, while its steady state performance was considerably worse than the LMS. Never the less, the NLMS is always the favorable choice of algorithm for fast convergence speed and for non-stationary input.

$$
h(t+1)=h(t)+\frac{\mu}{x^{T}(n) x(n)} e^{*}(n) x(n)
$$

Theoretically, the ranges of step size $(\alpha)$ for stable adaptation are from 0 to 2 ,however a more practical step size for NLMS is always less one unity .The above analyses provide a new method to obtain the optimal step size for stability and fast convergence in the mean convergence sense.

\section{The Normalized Least Mean Square (NLMS) Algorithm}

We may formulate the normalized LMS filter as a natural modification of the ordinary LMS filter. The normalized LMS filter is manifestation of the principle of minimal disturbance, which may be stated that the weight vector of an adaptive filter should be changed in a minimal manner, subject to a constraint imposed on the updated filter's output [7]. An issue of possible concern is that, in overcoming the gradient noise amplification problem associated with conventional LMS filter, the Normalized LMS filter introduces a problem of its own, namely that when the tap-input vector $u(n)$ is small, numerical difficulties may arise because then we have to divide by a small value 
for the squared norm $\|\mathrm{u}(\mathrm{n})\| 2$. To overcome this problem we modify the recursion of weighting factor as

$$
\mathrm{w}^{\wedge}(\mathrm{n}+1)=\mathrm{w}^{\wedge}(\mathrm{n})+\tilde{\mu} /\left[\delta+\|\mathrm{u}(\mathrm{n})\| \rrbracket \wedge 2 \mathrm{u}(\mathrm{n}) \mathrm{e}^{*(n)}\right.
$$

Whereas $\delta>0$

\section{Derivation of the Kalman based LMS (KLMS) Algorithm}

The Kalman filter can be used in adaptive filtering by making a number of correspondences. The adaptive filtering problem is reformulated as a state estimation problem, where the state vector corresponds to the filter coefficients vector. Since the state estimate is the state that minimizes the square of the error at each coefficient, it will also minimize the output error of the filter [6]. The optimal filter variation in time is modelled as a Markov model with white noise input, $\mathbf{n}(n)$, and state transition matrix, $\mathbf{F}(n)=\lambda \mathbf{I}$ with $\lambda$ close to one. The measured signal $d(n)$ is related to the state through the reference signal vector $\mathbf{u}(n)$ plus an additional measurement noise $v(n)$. This is summarized in Table II.

\section{Table I.}

\section{Correspondences from the Kalman Filter Variables ToAdaptive Filter Variables (NLMS)}

\begin{tabular}{|l|l|}
\hline Kalman & \multicolumn{1}{|c|}{$\begin{array}{c}\text { Kalman } \\
\text { LMS }\end{array}$} \\
\hline $\mathrm{z}(\mathrm{n})$ & $\mathrm{d}(\mathrm{n})$ \\
$\mathrm{H}(\mathrm{n})$ & $\mathrm{u}(\mathrm{n})$ \\
$\bar{x}_{\mid \mathrm{n}}(\mathrm{n})$ & $\mathrm{w}(\mathrm{n})$ \\
$\sum_{x \mid n-1}(n)$ & $\sum_{w}(n)$ \\
$Q_{n n}(n)$ & $Q_{n n}(n)$ \\
$Q_{v v}(n)$ & $q_{n}(n)$ \\
$\mathbf{F}(\mathrm{n})$ & $\Lambda \mathrm{i}$ \\
& \\
\hline
\end{tabular}

The resulting algorithm is then,

$$
\begin{aligned}
\alpha(n) & =d(n)-u^{T}(n) w(n) \\
w(n+1) & =\lambda w(n)+\lambda \frac{\sum_{w}(n) u(n) \alpha(n)}{u^{T}(n) \sum_{w}(n) u(n)+q_{v}(n)} \\
\sum_{w}(n+1) & =\lambda^{2} \sum_{w}(n)+-\lambda^{2} \frac{\sum_{w}(n) u(n) u^{T}(n) \sum_{w}(n)}{u^{T}(n) \sum_{w}(n) u(n)+q_{v}(n)}+Q_{n n}(n)
\end{aligned}
$$

The signal $\alpha(n)$ is the same as $e(n)$ and is used to be consistent with the Kalman filter notation. The variance matrix $\Sigma_{\mathrm{w}}(n)$ can be made diagonal by carefully selecting the state noise autocorrelation matrix $\mathbf{Q}_{n n}(n)$ at each iteration. More, this can be done without changing the state noise total power, $\operatorname{tr}\left\{\mathbf{Q}_{n n}(n)\right\}$, where $\operatorname{tr}\{\}$ stands for the trace 
of the matrix. The procedure is not feasible if the state noise is to low.To do this one simply makes $\boldsymbol{\Sigma}_{w}(n)=\sigma_{w}^{2}(n) \mathbf{I}$ and $\operatorname{tr}\left\{\mathbf{Q}_{n n}(n)\right\}=N q_{n}(n)$ and apply the trace operator to (10). The resulting algorithm is the Kalman based LMS algorithm (KLMS) and is represented in Table III. Note that $\operatorname{tr}\left\{\mathbf{u}(n) \mathbf{u}(n)^{\mathrm{T}}\right\}=\mathbf{u}(n)^{\mathrm{T}} \mathbf{u}(n)$. The actual algorithm presented in Table III has been modified to allow complex signals. Namely, in the calculation of the power and in the coefficients update, $\mathbf{u}(n)^{*}$, the conjugate of $\mathbf{u}(n)$, is used in its place.

Table II.

Normalized LMS Based on the Kalman Filter, KLMS

Initialize
$\begin{gathered}\mathrm{w}(0)=\mathrm{w}_{0} \\ \sigma_{w}^{2}(o)=\sigma_{w 0}^{2}\end{gathered}$
$\quad$ Iterate from $n=0$ to $\ldots$
$\begin{aligned} P(n) & =u^{H}(n) u(n) \\ \alpha(n) & =d(n)-u^{T}(n) w(n) \\ w(n+1) & =w(n)+\frac{u(n)^{*} \alpha(n)}{P(n)+q_{v}(n) / \sigma_{w}^{2}(n)} \\ \sigma_{w}^{2}(n+1) & =\sigma_{w}^{2}(n)\left(1-\frac{P(n) / N}{P(n)+q_{v}(n) / \sigma_{w}^{2}(n)}\right)+q_{v}(\mathrm{n})\end{aligned}$

\section{Information form Kalman based LMS (IKLMS) Algorithm}

If the state noise is low or zero (10) can be written as,

$$
\sum_{w}^{-1}(n+1)=\sum_{w}^{-1}(n)+u(n) u^{T}(n) / q_{v}(n)
$$

The matrix $\sum_{w}^{-1}(n+1)$ can be approximated by a diagonal matrix if the reference signal autocorrelation is narrow [8]. Doing this and applying the trace operator results,

$$
\sigma_{w}^{-2}(n+1)=\sum_{w}^{-1}(n)+p(n) / N q_{v}(n)
$$

by defining the total power up to (but not counting) time $n, P_{T}(n)$, by the equation,

$$
P_{T}(n+1)=P_{T}(n)+P(n)
$$

one can prove that,

$$
\sigma_{w}^{-2}(n)=\left(P_{T}(n)+N q_{v}(n) / 2 \sigma_{w 0}^{2}\right) / N q_{v}(n)
$$

with, $P_{T}(0)=0$, resulting in the algorithm presented in Table III.

Note that this algorithm is equivalent to the KLMS for the case $N=1$. 
Table III.

Information Form Kalman Based LMS (IKLMS

Initialize
$\mathrm{w}(0)=\mathrm{w}_{0}$
$P_{T}(0)=\sigma_{w 0}^{2}$
Iterate from $n=0$ to.
$\alpha(n)=d(n)-u^{T}(n) w(n)$
$P(n)=u^{H}(n) u(n)$
$w(n+1)=w(n)+\frac{u(n)^{*} \alpha(n)}{P(n)+P_{T}(n) / N+q_{v} / \sigma_{w 0}^{2}}$
$P_{T}(n+1)=P_{T}(n)+P(n)$

\section{Simplifications of the Algorithm (SIKLMS)}

If one is not interested in the initial convergence, then the algorithm in Table III can be simplified [9]. The coefficients estimation error standard deviation $\sigma_{w}^{2}(n)$ converges to a steady state value, resulting that $q_{v}(n) / \sigma_{w}^{2}(n)$ converges to,

$$
q_{v}(\infty) / \sigma_{w}^{2}(\infty)=\mathrm{p} / 2\left(-1+1+\sqrt{4 q_{v} / N P q_{v}}\right)
$$

This can be used in place of $q_{v}(n) / \sigma_{w}^{2}(n)$. The algorithm in IV can also be simplified. The time varying quantity $P_{T}(n+1)$ is replaced by an estimate of its value at time $M$, resulting in,

$$
w(n+1)=w(n)+\frac{u(n)^{*} \alpha(n)}{\left(\frac{N+M-1}{N}\right) u^{H}(n) u(n)+q_{v} / \sigma_{w 0}^{2}}
$$

We call this algorithm the Simplified Information Form Kalman LMS (SIKLMS). The quantity $M$ is the step sample time.

\section{Simulation Results:}

To show that SIKLMS algorithms are really effective in critical situations, the method has been validated using several ECG recordings with a wide variety of wave morphologies from MIT-BIH arrhythmia database. The convergence characteristics of various algorithms are shown in Figure 2. MIT-BIH arrhythmia ECG recordings used as reference signal and real noise are obtained MIT-BIH Normal Sinus Rhythm Database (NSTDB). 


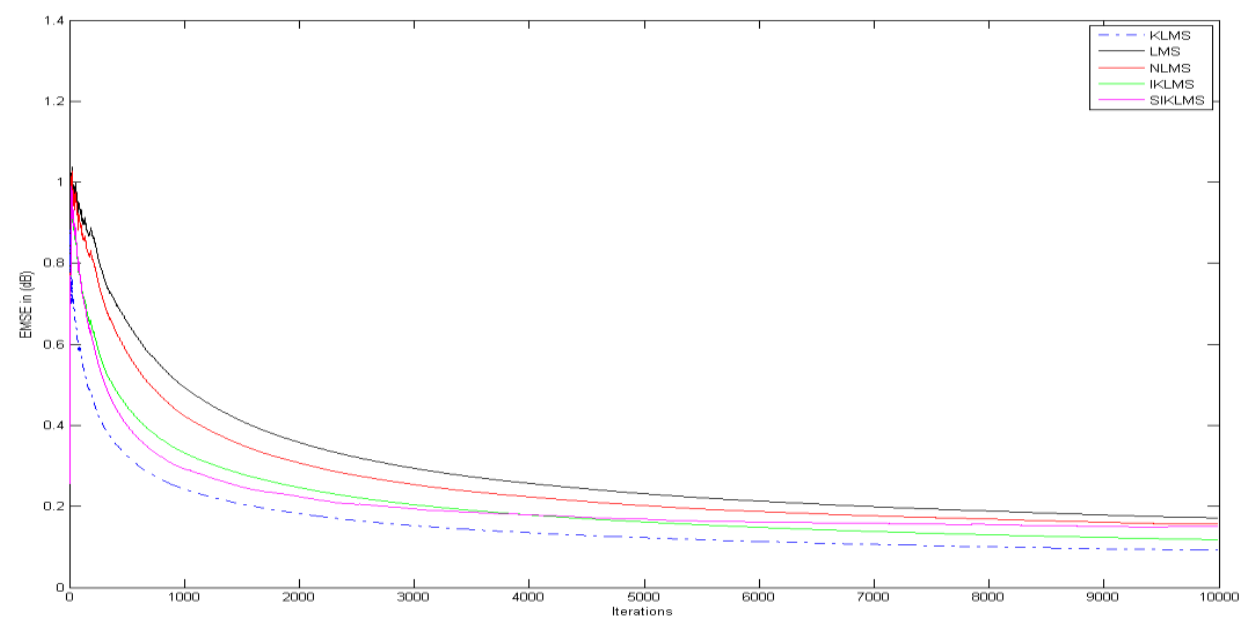

Figure 2. Convergence Characteristics of Various Algorithms

The Baseline Wander noise is added to the ECG signal shown in Figure 3. For evaluating the performance of the proposed SIKLMS adaptive filter considered the performance of LMS, NLMS, KLMS, IKLMS algorithms.

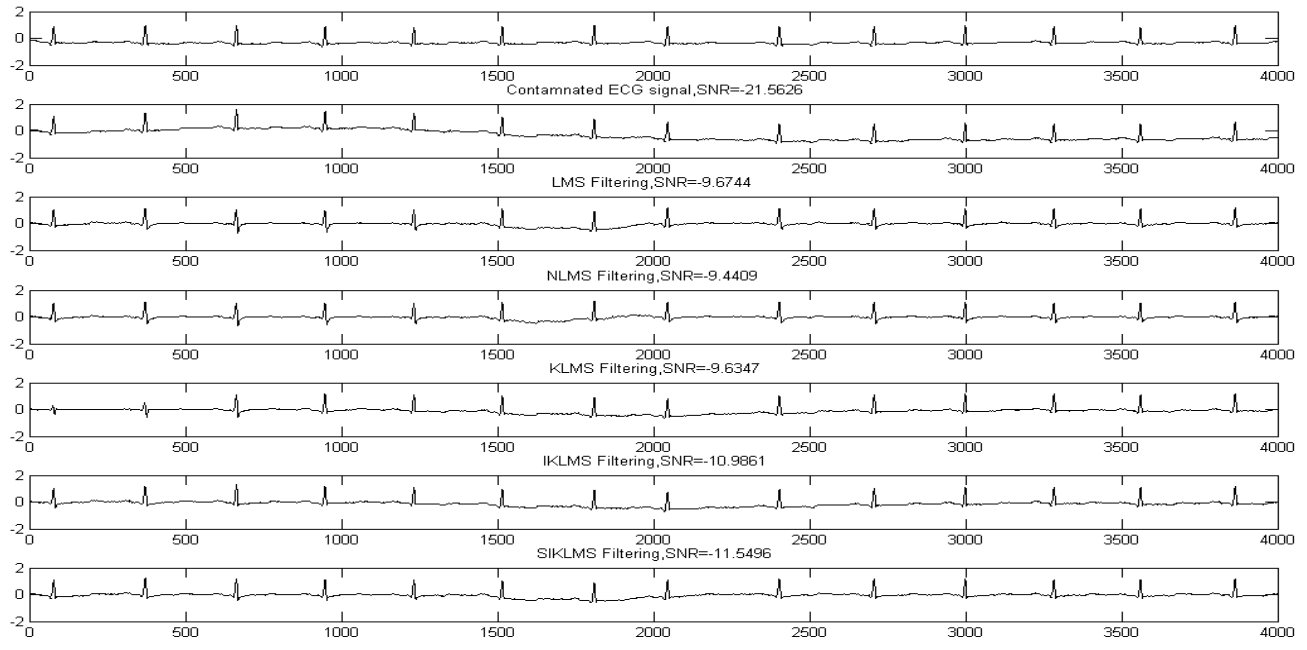

Figure 3. (a) ECG Signal,(b)Contaminated ECG Signal with Baseline Wander Noise,(c)Denoised ECG Signal with LMS Algorithm,(d) Denoised ECG Signal with NLMS Algorithm,(e) Denoised ECG Signal with KLMS Algorithm(f) Denoised ECG Signal with IKLMS Algorithm (g) Denoised ECG Signal with SIKLMS Algorithm

The ECG signal contaminated with Baseline Wander (BW) noise is applied as primary input to the adaptive filter of Fig.1. The real BW is given as reference signal. The simulation results shown in Fig 3 indicate the reduction of BW noise gradually. 


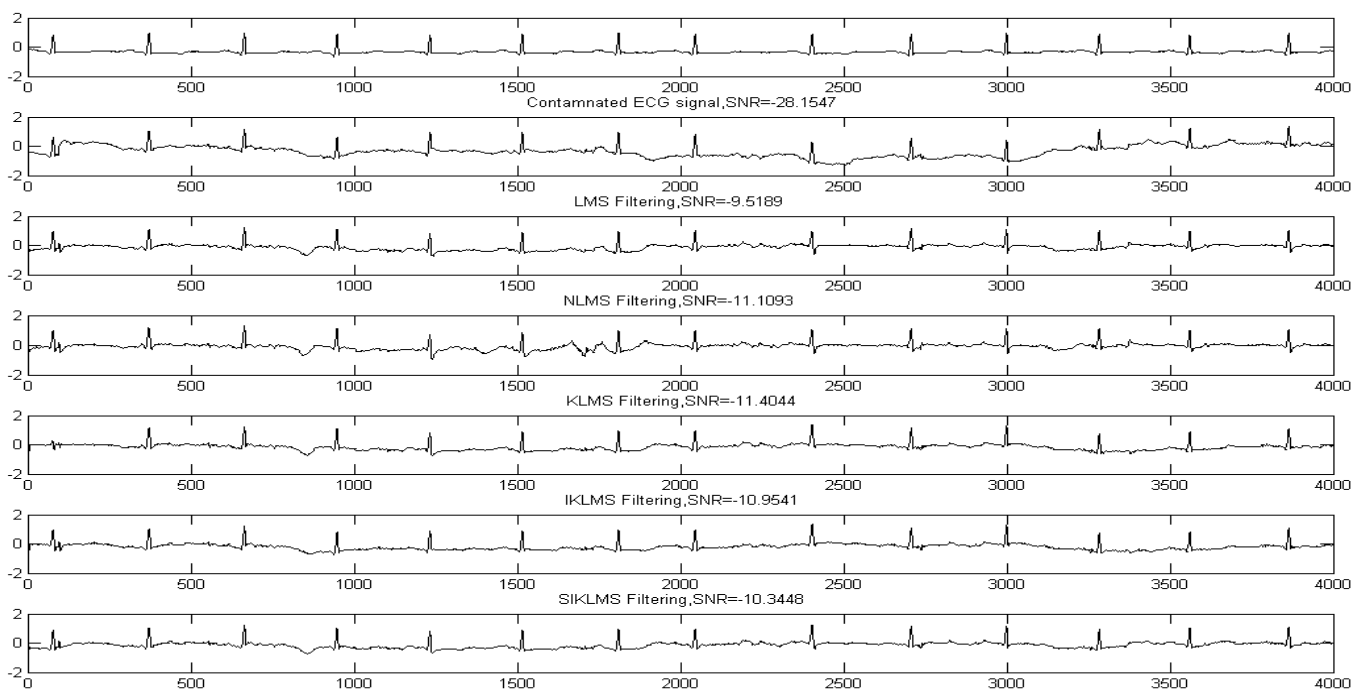

Figure 4. (a) ECG Signal,(b)Contaminated ECG Signal with Electrode Motion Noise,(c)Denoised ECG Signal with LMS Algorithm,(d) Denoised ECG Signal with NLMS Algorithm,(e) Denoised ECG Signal with KLMS Algorithm(f) Denoised ECG Signal with IKLMS Algorithm (g) Denoised ECG Signal with SIKLMS Algorithm

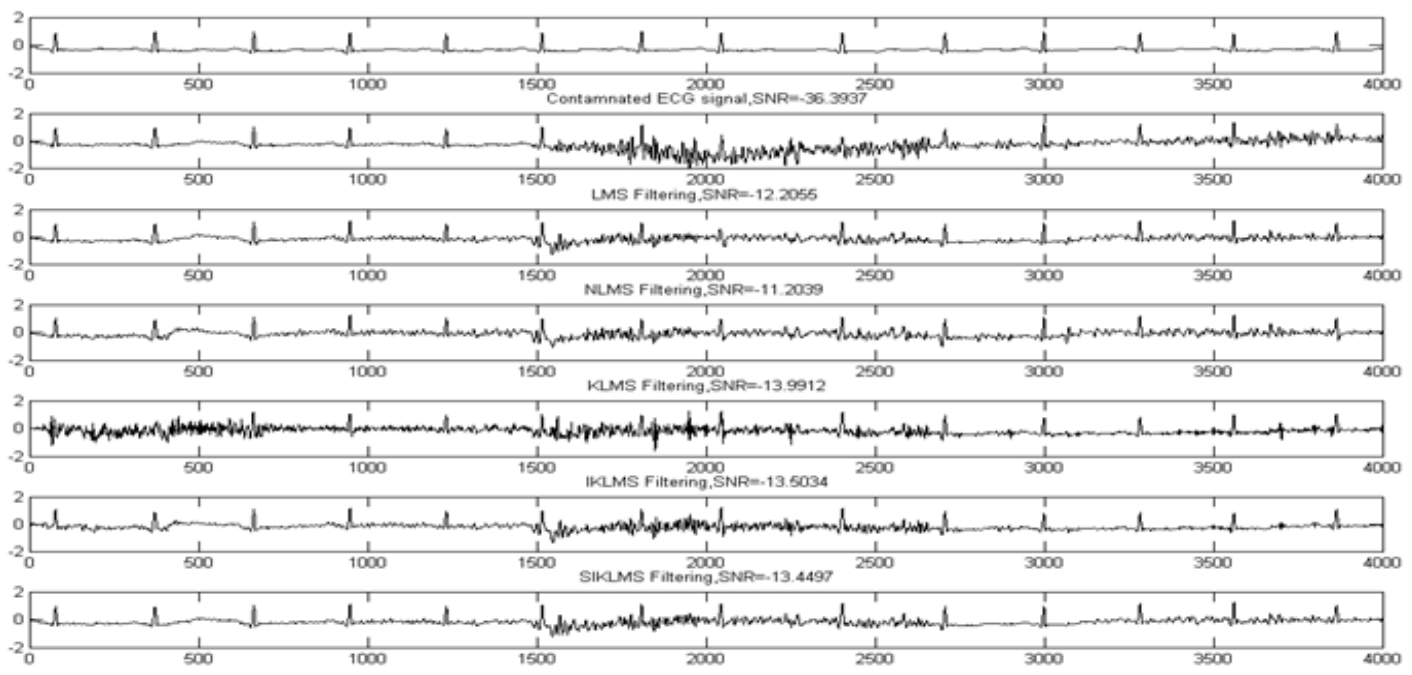

Figure 5. (a) ECG Signal,(b)Contaminated ECG Signal with Muscle Artifact,(c)Denoised ECG Signal with LMS Algorithm,(d) Denoised ECG Signal with NLMS Algorithm,(e) Denoised ECG Signal with KLMS Algorithm(f) Denoised ECG signal with IKLMS Algorithm (g) Denoised ECG Signal with SIKLMS Algorithm

We use a generated pure ECG signal with electrode motion artifact (EM) added, where EM is taking by a MIT-BIH database. The ECG signal contaminated with EM is given as input to the adaptive filter. The EM noise is given as reference signal. Output of the filter is the required to get better resolution ECG signal shown in Fig 4. In order to observe the filtering 
performance in the presence of non-stationary noise, muscle artifact (MA) was taken from the MIT-BIH Noise Stress Test Database. These muscle artifacts obscure the EEG and complicate the interpretation of the EEG or even make the interpretation unfeasible. Here the SIKLMS algorithm shows the better convergence results over all previous algorithms

\section{Conclusion}

In this paper we presented the performance evaluation of the SIKLMS adaptive algorithm noise removal from contaminated ECG over nonblind algorithms like LMS and NLMS. For this, the input and the desired response signals are properly chosen in such a way that the filter output is the best least squared estimate of the original ECG signal. The proposed SIKLMS algorithm shows the weight update formula and thus pushes up the speed over the respective LMS based realizations. Our simulations, however, confirm that the SNR of the KLMS algorithm gives better result over the other algorithms except BW Artifact as shown in below table v and SIKLMS algorithm gives better convergence, low complex over other algorithms.

\section{Table IV. SNR for Different Algorithms}

\begin{tabular}{|c|c|c|c|}
\hline Adaptive Algorithm & SNR for BW Artifact ( in dB) & SNR for EM Artifact ( in dB) & SNR for MA Artifact ( in dB) \\
\hline LMS & 9.67 & 9.51 & 12.20 \\
\hline NLMS & 9.44 & 11.10 & 11.20 \\
\hline KLMS & 9.63 & 11.40 & 13.99 \\
\hline IKLMS & 10.98 & 10.95 & 13.50 \\
\hline SIKLMS & 11.54 & 10.34 & 13.44 \\
\hline
\end{tabular}

\section{References}

[1] H. C. Y. Gu, K. Tang and W. Du, "Modifier formula on mean square convergence of LMS algorithm", Electronics Letters, vol. 38, no. 19, (2002) September, pp. 1147-1148.

[2] S. Haykin, “Adaptive Filter Theory”, Prentice-Hall, Inc., (1996).

[3] Y. Gu, K. Tang, H. Cui and W. Du, "Modifier formula on mean square convergence of LMS algorithm", Electronics Letters, vol. 38, no. 19, (2002) September, pp. 1147-1148.

[4] O. Sayadi and M. B. Shamsollahi, "ECG denoising and compression using a modified extended kalman filter structure", IEEE Trans. Biomed. Eng, vol. 55, no. 9, (2008), pp. 2240-2248.

[5] W. Zhang, T. Ma and L. Ge, "Enhancement of ECG signals by multi resolution sub band filter", 2nd International Conference on Bioinformatics and Biomedical Engineering, ICBBE 2008, China, (2008).

[6] J. Wang and Z. Li, "An ECG segmentation model used for signal generator", 2nd International Conference on Innovative Computing, Information and Control, ICICIC '07, Japan, (2007).

[7] N. V. Thakor and Y.-S. Zhu, "Applications of adaptive filtering to ECG analysis: noise cancellation and arrhythmia detection”, IEEE Transactions on Biomedical Engineering, vol. 38, no. 8, (1991), pp. 785-794.

[8] B. Farhang-Boroujeny, "Adaptive Filters- Theory and Applications", John Wiley and Sons. Chichester, UK, (1998).

[9] P. E. McSharry, G. D. Clifford, L. Tarassenko and L. A. Smith, "A dynamical model for generating synthetic electrocardiogram signals", IEEE Transactions on Biomedical Engineering, vol. 50, no. 3, (2003), pp. 289294.

[10] S. Makino and Y. Kaneda, "A new RLS algorithm based on the variation characteristics of room impulse response", IEEE International Conference, Japan, (1994).

[11] Y. Lu, J. Yan and Y. Yam, "Model-based ECG denoising using empirical mode decomposition", IEEE International Conference on Bioinformatics and Biomedicine, USA, (2009). 


\section{Authors}

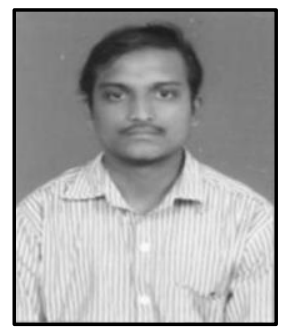

Y. Murali Krishna, He received his M.Tech degree from JNTUK, A.P, and India in 2011. Presently he is working as a Assistant professor, in Department of Electronics and Communication Engineering, Narasaraopeta Engineering College, Guntur, A.P, India. His current areas of research interests include Communication and Radar systems, Smart antennas.

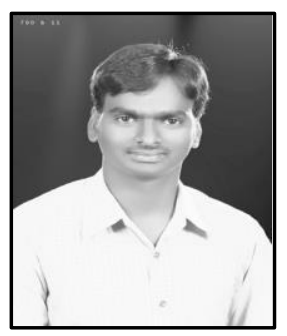

N. Sayedu khasim, He received his M.Tech degree from ANU, A.P, and India in 2010. Presently he is working as a Assistant professor, in Department of Electronics and Communication Engineering, Narasaraopeta Engineering College, Guntur, A.P, India. His current areas of research interests include Communication and Radar systems, Digital signal processing.

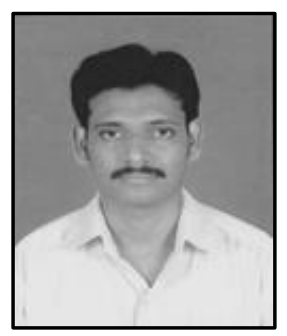

V. Naveen Raja, He received his M.Tech degree from JNTU Kakinada, A.P, and India in 2013. Presently he is working as a Assistant professor, in Department of Electronics and Communication Engineering, Narasaraopeta Engineering College, Guntur, A.P, India. His current areas of research interests include VLSI, Embedded Systems, Digital signal processing. 